\title{
Microencapsulación de Antocianinas de Berenjena (Solanum melongena L.) mediante Secado por Aspersión y Evaluación de la Estabilidad de su Color y Capacidad Antioxidante
}

\author{
Guillermo Arrazola, Irina Herazo y Armando Alvis \\ Grupo Investigación Procesos y Agro Industria de Vegetales. Programa Ingeniería de Alimentos. \\ Universidad de Córdoba. Colombia. (e-mail): guillermo.arrazola@ua.es
}

Recibido Oct. 15, 2013; Aceptado Nov. 28, 2013; Versión final recibida Dic. 18, 2013

\begin{abstract}
Resumen
Se extrajo antocianinas de la cáscara de berenjena (Solanum melongena L.), con diferentes concentraciones de etanol acidificado al 1\%. Los extractos de antocianinas se microencapsularon evaluando la influencia de las condiciones de secado por aspersión en las propiedades fisicoquímicas de los polvos. Las variables fueron temperatura del aire de entrada $\left(170\right.$ y $\left.180^{\circ} \mathrm{C}\right)$ y la concentración de maltodextrina (15, 20 y $30 \%)$. Se realizaron análisis de contenido de antocianinas, parámetros de color, capacidad antioxidante, humedad, actividad de agua, higroscopicidad, solubilidad, densidad, y espectrofotometría electrónica de barrido. La más alta concentración de antocianinas a partir de cáscara de berenjena $(115 \mathrm{mg} / 100 \mathrm{~g})$ se obtuvo con $53 \%$ etanol, por $3 \mathrm{~h}$ y a $29^{\circ} \mathrm{C}$ de temperatura. Durante la microencapsulación por secado por aspersión se observó una influencia estadísticamente significativa de la temperatura y el porcentaje de maltodextrina empleado en la microencapsulación $(p<0.05)$ sobre la mayoría de las propiedades fisicoquímicas de los extractos.
\end{abstract}

\section{Anthocyanins Microencapsulation of Eggplant (Solanum melongena L.) and Evaluation of Color Stability and Antioxidant Capacity}

\begin{abstract}
Anthocyanins from eggplant peels (Solanum melongena L.) were extracted with different concentrations of ethanol acidified at $1 \%$. Anthocyanin extracts were microencapsulated evaluating the influence of the spray-drying conditions on the physicochemical properties of the powders. Variables were the inlet air temperature $\left(170\right.$ to $180{ }^{\circ} \mathrm{C}$ ) and the concentration of maltodextrin $(15,20$ and $30 \%)$. Analysis of anthocyanin content, color parameters, antioxidants, humidity, water activity, hygroscopicity, solubility, density, and scanning electron spectrophotometry, were performed. The highest concentration of anthocyanins from eggplant peels $(115 \mathrm{mg} / 100 \mathrm{~g})$ was obtained with $53 \%$ ethanol, $3 \mathrm{~h}$ and $29{ }^{\circ} \mathrm{C}$ of temperature. During microencapsulation by spray drying, a statistically significant influence of the temperature and of the percentage of maltodextrin used in the microencapsulation $(p<0.05)$ on most physicochemical properties of the extracts was observed.
\end{abstract}

Keywords: eggplant, anthocyanins, microencapsulation, temperature, maltodextrin 


\section{INTRODUCCIÓN}

Las antocianinas son glucósidos de antocianidinas, pertenecientes a la familia de los flavonoides, compuestos por dos anillos aromáticos $\alpha$ y $\beta$ unidos por una cadena de 3 carbonos (Garzón, 2008). Estos pigmentos se encuentran ampliamente distribuidos en frutas y flores, ofreciendo atractivos colores, como anaranjado, rojo y azul (Tonon et al., 2010). El interés por los pigmentos antocianos en investigaciones científicas se han incrementado en los últimos años, debido no sólo al color que confieren a los productos que las contienen sino a su probable papel en la reducción de las enfermedades coronarias, cáncer, diabetes, efectos antiinflamatorios, mejoramiento de la agudeza visual y comportamiento cognitivo; estos efectos preventivos y terapéuticos, están principalmente asociados con sus propiedades antioxidantes (Longo y Vasapollo, 2004; Garzón, 2008). Por lo tanto, además de su papel funcional, las antocianinas como pigmentos naturales se constituyen en agentes potenciales para la obtención de productos nutraceúticos.

La berenjena es de color verdoso, negro, morado, blanco, blanco jaspeado de morado, lila u oscuro que suele tener forma redondeada, periforme u ovalada y de variada longitud (García et al., 2003; FAO, 2007). Contiene ácido ascórbico y compuestos fenólicos que le confieren un gran poder antioxidante, principalmente asociado a algunos efectos terapéuticos positivos (Noda et al., 2000; Nisha et al., 2009; Todaro et al., 2009; Singh et al., 2009). Algunos autores han obtenido antocianinas de cáscara de berenjena como la antocianina acilada nasunina (Noda et al., 2000; Ichiyanagi et al., 2006; Calogero y Di Marco, 2008), delfinidina-3-rutinósido (Todaro et al., 2009) y delfinidina 3- rutinósido -5- galactósido, delfinidina -3rutinósido-5- glucósido, delfinidina -3- glucósido y delfinidina -3-rutinósido (Wu y Prior, 2005).

Los antocianos tienen baja estabilidad, debido a la sensibilidad, cambios de $\mathrm{pH}$, temperatura, luz, oxigeno, entre otros factores, los cuales se convierten en la principal limitación de estos pigmentos para ser aplicados como sustitutos potenciales de los colorantes artificiales en alimentos (Owusu, 2005; Poo, 2005; Castañeda et al., 2009; Olaya et al., 2009). Las aplicaciones de técnicas de microencapsulación han ido incrementándose en la industria de los alimentos, debido a la protección de los materiales encapsulados frente a diferentes factores que afectan su vida útil, permitiendo, mantener su estabilidad y viabilidad en el tiempo (Ribeiro y Stringheta, 2006; Olaya et al., 2009). Muchos autores han trabajado con diferentes técnicas de microencapsulación de antocianinas con la finalidad de ofrecer la protección y estabilidad de estos pigmentos, mejorando de esta forma su utilización como ingredientes alimenticios (Provenzi et al., 2006; Ribeiro y Stringheta, 2006; Ersus y Yurdagel, 2007; Valduga et al., 2008; Olaya et al., 2009; Tonon et al., 2010). En comparación con otros métodos, el secado por aspersión proporciona una eficiencia de encapsulación relativamente alta. En un proceso de secado por aspersión se deben tener en cuenta algunos parámetros como: temperatura de entrada y salida del aire de secado, el flujo de alimentación del producto a secar, la concentración de agente encapsulante y el acondicionamiento de la materia prima, entre otros (García et al., 2004).

El objetivo de esta investigación, fue microencapsular las antocianinas de cáscara de berenjena, mediante la microencapsulación por secado por aspersión con maltodextrina, evaluando la influencia de las condiciones de secado por aspersión en las propiedades fisicoquímicas y antioxidantes de los extractos y su estabilidad mediante HPLC.

\section{MATERIALES Y MÉTODOS}

\section{Obtención de antocianinas a partir de cáscara de berenjena}

Se realizó la recolección de berenjenas (Solanum melongena L.) morada negra cultivada en el departamento de Córdoba. La recolección de las berenjenas se realizó en la fase de maduración comercial. Para la extracción de antocianinas de la cáscara de berenjena, las muestras fueron peladas y cortadas empleando un cuchillo de acero inoxidable según (Todaro et al., 2009).

\section{Extracción y cuantificación de antocianinas.}

Las muestras de cáscara de berenjena previamente acondicionadas se sometieron al proceso de extracción en una relación 1:10 ( $1 \mathrm{~g}$ de cáscara de berenjena con $10 \mathrm{~mL}$ de etanol acidificado con ácido clorhídrico $(\mathrm{HCl})$ al $1 \%$ ). Una vez finalizada la obtención, la mezcla se filtró en un embudo Buchner y el sólido recuperado fue lavado con el solvente etanol acidificado. Luego se combinaron los sobrenadantes de las filtraciones. Para llevar a cabo la extracción de las antocianinas de la berenjenas se emplearon las siguientes condiciones de extracción: utilizando como solvente etanol acidificado (Etanol desde el 50 - $90 \%$ v/v), temperatura de extracción ( 30 a $60^{\circ} \mathrm{C}$ ) y tiempo de extracción (4 - $12 \mathrm{~h}$ ) (Olaya et al. 2009; Todaro et al. 2009; Martínez 2009). 


\section{Microencapsulación de antocianinas por secado por aspersión con maltodextrina}

Antes de iniciar la microencapsulación por aspersión, se prepararon mezclas de maltodextrina y extractos (DE) al 15, 20 y $30 \%$, maltodextrina añadida al extracto. Se empleó un secador por aspersión Modelo B290 (Büchi). Las condiciones del proceso que se manejaron fueron temperatura de entrada de 170 y $180 \stackrel{\circ}{ }$; velocidad de aspiración $75 \%$ (30 m³ / h aproximadamente). (Aguilera-Ortiz et al. 2012; Da silva et al. 2010). El rendimiento en peso obtenido tras el secado por atomización se calculó a partir de las determinaciones del peso de polvo obtenido, de acuerdo con la ecuación 1:

Rendimiento en peso $=($ gramos obtenidos $/$ gramos en la mezcla inicial $) * 100$

Los gramos en la mezcla inicial se calcularon a partir de los gramos de material y el volumen de extracto utilizados como material de partida. Los extractos obtenidos fueron sometidos a diferentes análisis:fisicoquímico de los microencapsulados, contenido de antocianinas, poder antioxidante de polvos microencapsulados y color de los polvos secados por atomizado.

\section{Análisis fisicoquímico de los microencapsulados}

Para la humedad se tomó $1 \mathrm{~g}$ de pigmentos encapsulados, se colocó en una cápsula de porcelana a 105 ㄷ en una estufa de aire forzado, hasta alcanzar peso constante. La humedad de los microencapsulados se determinó por secado y diferencia de peso de acuerdo al método 934.06 de la AOAC. La actividad de agua de los polvos se determinó mediante un equipo Novasina (Lab Master), a $25^{\circ} \mathrm{C}$ a un tiempo de observación de 2 minutos. Para la higroscopicidad se tomó $1 \mathrm{~g}$ de los polvos, se colocaron en capsulas de Petri a $25^{\circ} \mathrm{C}$, que se introdujeron en una cámara que contenía una solución saturada de $\mathrm{NaCl}(75,4 \%$ de humedad relativa). Después de 1 semana se pesaron las muestras. Se expresaron los resultados como $\mathrm{g}$ de humedad por $100 \mathrm{~g}$ de sólidos secos (g /100 g) (Cai y Corke, 2000; Ersus y Yurdagel, 2007). Para evaluar la solubilidad a $100 \mathrm{~mL}$ de agua destilada se añadió $1 \mathrm{~g}$ de polvo, se agitó manualmente hasta solubilizar toda la muestra, luego se realizó una centrifugación a $3000 \mathrm{rpm}$ durante $10 \mathrm{~min}$. Se tomó una muestra representativa de $25 \mathrm{~mL}$ del sobrenadante y se pasó a capsulas de Petri. Finalmente se procedió a secar la muestra en una estufa a $105^{\circ} \mathrm{C}$ por $5 \mathrm{~h}$. La solubilidad (\%) es calculada por diferencia de peso (Ochoa et al., 2011). La densidad aparente de los polvos se midió pesando $2 \mathrm{~g}$ de muestra, las cuales fueron colocadas en una probeta graduada de $10 \mathrm{~mL}$. La densidad aparente se calculó dividiendo la masa de polvo por el volumen ocupado en el cilindro (Cai y Corke 2000). La morfología de las partículas se evaluó por microscopía electrónica de barrido (SEM). Los polvos fueron unidos a una cinta adhesiva de doble cara montado sobre talones de SEM, recubierto con 3-5 mA oro / paladio al vacío y se examinaron con un microscopio electrónico de barrido JEOL® modelo JSM 6490 LV, operado en el modo de alto vacío.

\section{Análisis de contenido de antocianinas por cromatografía líquida de alta resolución (HPLC)}

Se cuantificó el contenido de antocianinas del extracto seleccionado para el proceso (Todaro et al, 2009), de microencapsulación y de los polvos microencapsulados por (HPLC); utilizando un Cromatógrafo (Accela 600 Thermo Scientific), detector con arreglo de diodos. Se empleó una columna RP-18, con un volumen de muestra de $1 \mu \mathrm{L}$, una longitud de onda de detección de $525 \mathrm{~nm}$, a $25^{\circ} \mathrm{C}$ y utilizando un flujo de la fase móvil de $200 \mu \mathrm{Lmin}^{-1}$. Se empleó como fase móvil acetonitrilo (\% Fase A) y agua conteniendo $1 \%$ de ácido fórmico (\% Fase B) (Durst y Wrolstad 2001; Todaro et al. 2009).

\section{Determinación de poder antioxidante de polvos microencapsulados}

La actividad antioxidante de los polvos se midió empleando el método ABTS* (6-sulfonato-3etilbenzotiazolina), las mediciones de absorbancia se realizaron a $732 \mathrm{~nm}$ empleando un espectrofotómetro (Génesis 20). Para la determinación se emplearon $10 \mu \mathrm{L}$ del extracto diluido en DMSO (Dimetilsufoxido) y $990 \mu \mathrm{L}$ de la solución del radical $\mathrm{ABTS}^{\star}$. Luego de $30 \mathrm{~min}$ de reacción a temperatura ambiente y en la oscuridad, se leyó el cambio en la absorbancia de la muestra. Los resultados se expresaron como IC50, el cual corresponde a la concentración necesaria para inhibir el $50 \%$ de la absorbancia del radical. También se calcularon valores TEAC mediante la construcción de una curva patrón usando diferentes concentraciones de TROLOX® (ácido 6-hidroxi -2,5,7,8- tetrametil-2- cromanocarboxílico) y Ácido ascórbico VEAC, (Martínez, 2009).

Análisis de color de los polvos secados por atomizado

Los parámetros de color $L^{*}$, a *, $b$ * $C^{*}$ (Saturación de color) y ${ }^{\circ} \mathrm{H}$ (Angulo de tono) se midieron con un colorímetro Colorflex EZ 45 (HunterLabß). El colorímetro se calibró con un plato de cerámica estándar de color verde y blanco antes de su lectura. 


\section{Análisis estadístico de los datos}

En los resultados obtenidos, se analizaron los errores experimentales, se evaluaron los supuestos de aleatoriedad, linealidad, normalidad y homogeneidad de varianzas. Comprobados los supuestos, a las variables respuesta se les realizaron los análisis de varianzas, test de diferencias de medias (Tukey) y ajuste de regresiones con significancia del $5 \%$. Estos análisis se realizaron en el programa SAS versión 9.2. En la evaluación de la estabilidad de los extractos de antocianinas de berenjena en bebidas, se analizaron los errores experimentales, se evaluaron los supuestos de aleatoriedad, linealidad, normalidad y homogeneidad de varianzas. Comprobados los supuestos, a las variables respuesta se les realizaron los análisis de varianzas, test de diferencias de medias (Tukey) y ajuste de regresiones con significancia del $5 \%$. Estos análisis se realizaron en el programa SAS versión 9.2.

\section{RESULTADOS Y DISCUSIÓN}

\section{Microencapsulación de antocianinas}

Al extracto se le adicionó maltodextrina como agente encapsulante en concentraciones del 15, 20 y $30 \%$. El extracto de antocianinas empleado presentó valores alrededor de $55 \mathrm{mg} / 100 \mathrm{~g}$ de antocianinas. Durante el proceso de secado por atomización se emplearon las temperaturas de entrada de aire de 170 y $180{ }^{\circ} \mathrm{C}$, obteniéndose polvos secos de color rosado. La caracterización fisicoquímica de los polvos microencapsulados a diferentes tratamientos, (figura 1). Los valores medios de las variables fisicoquímicas humedad, actividad de agua y solubilidad presentaron diferencias significativas $(p<0,05)$, para los factores temperatura de entrada del aire y concentración de agente encapsulante (maltodextrina). Sin embargo la densidad y la higroscopicidad no presentaron diferencias significativas con respecto al factor temperatura de entrada del aire. Existe interacción significativa entre las dos variables independientes para todos los parámetros fisicoquímicos estudiados en los polvos. El contenido de humedad para el extracto microencapsulado fue significativamente mayor en los polvos sometidos a temperaturas de entrada de 170 ${ }^{\circ} \mathrm{C}$, siendo evidente que en la misma concentración de agente encapsulante, la temperatura de secado más alta produjo el menor contenido de humedad del producto seco. Este comportamiento ha sido observado por Bakowska-Barczak y Kolodziejczykb (2011), Ersus y Yurdagel (2007), Jittanit et al. (2011) y Silva et al. (2013) los cuales estudiaron el efecto de la temperatura de entrada de aire de secado sobre la humedad de extractos microencapsulados de grosella negra, zanahoria negra, tamarindo y Jaboticaba respectivamente. A temperaturas más altas de entrada de aire, existe un gradiente de temperatura mayor entre la alimentación atomizada y el aire de secado, lo que resulta en una mayor fuerza motriz para la evaporación del agua y por lo tanto la producción de polvos con menor contenido de humedad (Tonon et al., 2008; Phisut, 2012). Asimismo a medida que se aumentó la concentración de agente encapsulante en el flujo de alimentación, se disminuyó el contenido de humedad de los polvos. Resultados similares observaron RuizCabrera et al. (2009) y Ahmed et al. (2010) en donde el contenido de humedad disminuyó a medida que aumentó la concentración de maltodextrina en muestras de batata morada y maracuyá respectivamente.

Existe un efecto de interacción significativo entre las variables independientes, el menor contenido de humedad (3.43\%) de los polvos se obtuvo con la temperatura de $180{ }^{\circ} \mathrm{C}$ y $30 \%$ de sólidos en la alimentación. Estudios anteriores revelan que la humedad de microcápsulas con maltodextrina presentaron valores entre 4.04 y $5.5 \%$, varios autores (Escalona, 2004; Ersus y Yurdagel, 2007; Valduga et al., 2008; Fang y Bhandari, 2011). Bakowska-Barczak y Kolodziejczykb (2011) reportaron que el contenido de humedad varió desde 1.8 hasta 3.9 en extractos de antocianina de Grosella negra microencapsulados con maltodextrina. La actividad de agua de las antocianinas microencapsuladas mostró una influencia significativa de la temperatura de entrada, siendo menor a $180{ }^{\circ} \mathrm{C}$. Además el tratamiento con agente encapsulante del $15 \%$ exhibió la mayor actividad de agua, mientras que los del 20 y $30 \%$ no difieren significativamente entre sí. De esta manera se observa un comportamiento similar del contenido de agua con respecto al factor temperatura y concentración de agente encapsulante, siendo obtenida la menor actividad de agua (0.25) con $20 \%$ de sólidos y $180^{\circ} \mathrm{C}$. Los valores de actividad de agua de los polvos microencapsulados son similares a los reportados por Valduga et al. (2008) (0.266); Fang y Bhandari (2011) (0.215); Jittanit et al. (2011) (0.260 y 0.342). La higroscopicidad de los polvos microencapsulados no presentó diferencias significativas entre las medias de los valores de los tratamientos a diferentes temperaturas. Cai y Corke (2000) observaron que la humedad higroscópica de polvos de betacianina secados por aspersión con maltodextrina (15 DE) varió de 45.4 a $49.2 \mathrm{~g} / 100 \mathrm{~g}$, cuando la temperatura aumentó desde 150 a $210{ }^{\circ} \mathrm{C}$, presentando la mayoría de los tratamientos diferencias en cuanto a la temperatura. Asimismo Silva et al. (2013), no encontraron diferencias significativas de los polvos microencapsulados con la temperatura del aire de entrada.

Se ha observado una relación inversa entre la higroscopicidad y el contenido de humedad de los polvos. Los polvos obtenidos a temperaturas de entrada de aire más altas son más higroscópicos debido al menor 
contenido de humedad en el polvo. Esto está relacionado con el gradiente de concentración de agua entre el producto y el aire circundante, que es ideal para polvos menos húmedos (Tonon et al., 2008; Phisut, 2012; Tee et al., 2012). Sin embargo esta relación inversa de la humedad y la higroscopicidad y por ende con la temperatura de entrada del aire no siempre se da, autores como Ruiz-Cabrera et al. (2009) observaron que la higroscopicidad y el contenido de humedad disminuían en el rango de temperaturas de 188 a $190{ }^{\circ} \mathrm{C}$, indicando además que el comportamiento general fue que el contenido de humedad y la higroscopicidad disminuyeron con las altas temperaturas. Caparino et al. (2012) también observó una pequeña relación directa entre el contenido de humedad de las muestras y la higroscopicidad. Por otro lado la higroscopicidad de las muestras varió significativamente con las concentraciones de maltodextrina, siendo los tratamientos con menor contenido de maltodextrina (15\%) los de mayor higroscopicidad y los del 20 y $30 \%$ no presentaron diferencias en cuanto a esta variable. La interacción de las dos variables independientes muestra interacción significativa, siendo el tratamiento con $20 \%$ de maltodextrina y $170{ }^{\circ} \mathrm{C}$ el polvo con menor higroscopicidad (25.35\%).
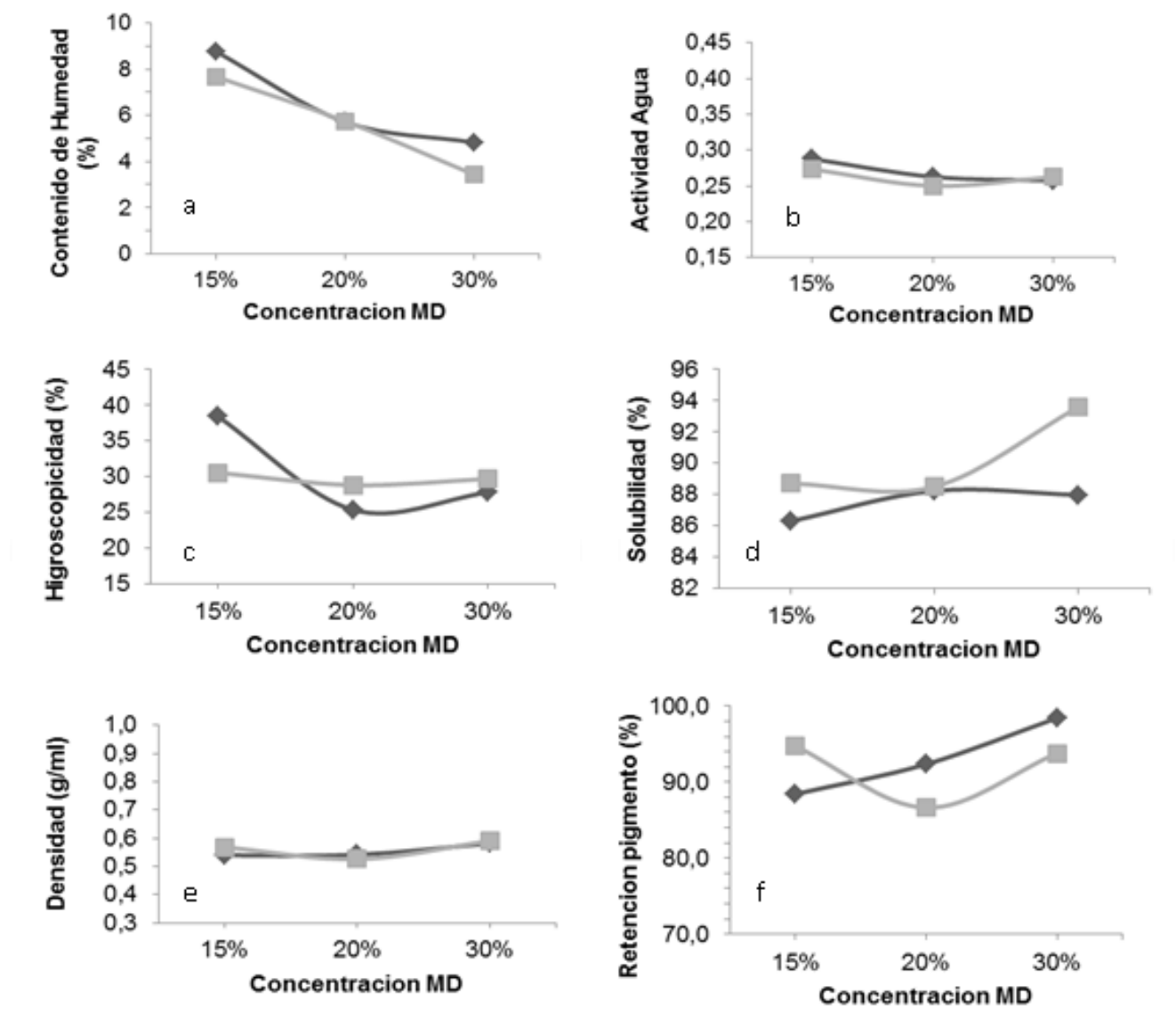

Fig. 1: Comportamiento de la Humedad (a), actividad de agua (b), higroscopicidad (c), solubilidad (d), densidad (e) y Retención del pigmento (f) de los polvos obtenidos a diferentes concentraciones de maltodextrina y a temperaturas de secado de $170^{\circ} \mathrm{C}(\square)$ y $180^{\circ} \mathrm{C}(\diamond)$.

Cai y Corke (2000), reportaron que las propiedades higroscópicas de los polvos fue significativamente menor $(44.6 \mathrm{~g} / 100 \mathrm{~g})$ cuando el flujo de alimentación tenía un $39.2 \%$ de solidos (Extracto de betacianina y maltodextrina $15 \mathrm{DE})$ que cuando se empleó una alimentación de $10 \%$ de solidos $(49.5 \mathrm{~g} / 100 \mathrm{~g})$. Así mismo Tonon et al. (2008) observaron un comportamiento similar, pues los valores más bajos de higroscopicidad se obtuvieron cuando se utilizaron las concentraciones más elevadas de maltodextrinas. Esto se debe al hecho de que la maltodextrina es un material con una baja higroscopicidad lo que sugiere que la maltodextrina es un eficiente agente auxiliar en la reducción de la higroscopicidad del material secado (Ahmed et al., 2010; Caparino et al., 2012). Los polvos microencapsulados mostraron que la solubilidad fue significativamente influida por la temperatura de entrada, siendo más solubles a medida que se incrementa la temperatura. Jittanit et al. (2011), observaron que la temperatura de secado tiene un efecto positivo sobre la solubilidad, debido a que las altas temperaturas del aire de secado por aspersión producen más porosidad de los polvos. La mayor porosidad da lugar a una mayor superficie específica de polvo, lo que resulta en un área de contacto más grande entre el polvo y el agua. Sin embargo Rivas (2010) observó que la solubilidad no varía con el aumento de la temperatura de salida del aire obteniendo valores entre el 75 y $76 \%$ en polvos de jugo de chirimoya microencapsulados con maltodextrina en un rango de temperatura de 
120 a $160{ }^{\circ} \mathrm{C}$. Además el tratamiento con agente encapsulante del $30 \%$ y $180{ }^{\circ} \mathrm{C}$ mostró la mayor solubilidad (93.61\%), mientras que los del 15 y $20 \%$ no difieren significativamente entre sí. Cano-Chauca et al. (2004) observaron que en la obtención de polvos de mango el tratamiento con maltodextrina presentó un alto grado de solubilidad, alcanzando valores superiores al $90 \%$. La maltodextrina es una de las sustancias más utilizadas como agente aditivo en la deshidratación de extractos y jugos por secado en atomizador debido a sus propiedades físicas, tales como alta solubilidad en agua (Cai y Corke, 2000; Cano-Chauca et al., 2004; Caparino et al., 2012). Ceballos (2008) reportó valores de solubilidad entre 84.8 y $91.5 \%$ para polvos deshidratados de frutas. Existe una interacción significativa $(P<0.05)$ entre la temperatura y la concentración de agente encapsulante. La figura 1d muestra que a medida que aumenta la concentración de agente encapsulante la solubilidad muestra incremento a $180{ }^{\circ} \mathrm{C}$ mientras que a $170{ }^{\circ} \mathrm{C}$ se alcanzaron valores menores de higroscopicidad que con $180^{\circ} \mathrm{C}$, para todos los niveles de concentración.

Para todos los niveles de concentración de maltodextrina, la densidad aparente fue significativamente diferente, observándose que esta variable fue mayor cuando se usó la concentración de 30\% de maltodextrina. Diversos autores han observado un comportamiento similar al microencapsular con maltodextrina por secado por aspersión pigmentos de betacianinas y extractos de granada (Cai y Corke, 2000; Miravet, 2009). Sin embargo Jittanit, et al. (2011) y Caparino et al. (2012) notaron que a mayor proporción de maltodextrina la densidad aparente disminuía. La densidad aparente del pigmento en polvo microencapsulado no presentó diferencia significativa con el aumento de temperatura del aire de secado (Figura 1e). Sin embargo algunos autores han encontrado que el aumento de la temperatura causa la reducción de la densidad aparente (Tonon et al., 2011; Phisut, 2012).

Según el análisis estadístico realizado la interacción entre la concentración de agente encapsulante y la temperatura fue significativa $(P<0.05)$ en la densidad de los polvos. Los valores de densidad obtenidos para los polvos microencapsulados coinciden con los reportados por Cai y Corke (2000) $(0,52-0,68 \mathrm{~g} / \mathrm{mL})$, Miravet (2009) (0.50 y $0.60 \mathrm{~g} / \mathrm{mL}$,), Ochoa et al. (2011) $(0.51$ a $0.61 \mathrm{~g} / \mathrm{mL})$. Sin embargo estos resultados son mayores a los reportados por Tonon et al. (2010) en microencapsulados de extractos de fruto de Acai (Euterpe oleracea Mart.) con maltodextrina $(0,37-0,39 \mathrm{~g} / \mathrm{mL})$. El tratamiento a temperatura de $180{ }^{\circ} \mathrm{C}$ y $30 \%$ de maltodextrina, fue el que presentó lo mejores parámetros fisicoquímicos, con menor contenido de humedad, menor higroscopicidad y mayor solubilidad, lo que lo hacen un extrto seco microencapsulado con buenas características de aplicación industrial.

\section{Contenido de antocianinas retenida en el extracto microencapsulado}

El contenido de antocianinas en los polvos secados por aspersión se determinó por HPLC, por el método de estándar externo. La figura 2a muestra el pico característico del estándar Delfinidina-3-rutinoside, mientras que en la figura $2 b$ y $2 c$ se pueden observar los picos para el extracto empleado en la microencapsulación y para los polvos secos por atomizado respetivamente.

Para la cuantificación de antocianinas en los polvos se elaboró una curva patrón a partir del estándar Delfinidina-3-rutinoside. En la curva de calibración con el estándar se obtuvo un $\mathrm{R}^{2}$ de 0.99 , ecuación de la curva, $\mathrm{Y}=\mathrm{m} \mathrm{a}+\mathrm{b}, \mathrm{a}=1,70917$ y $\mathrm{b}=-2,10308$. Para los picos identificados en el extracto de antocianinas, no se tuvieron en cuenta los que tenían áreas menores de $3 \%$ y una pobre resolución. De esta manera las dos principales antocianinas, correspondientes a los picos 2 y 3, representan aproximadamente el $16.7 \%$ y $80 \%$, respectivamente del área total del pico revelada a $520 \mathrm{~nm}$. Los tiempos de retención para las antocianinas correspondiente al pico 2 fueron para el extracto y para los polvos de 1.012 min, los cuales coincide con el pico identificado en el estándar. De acuerdo a los tiempos de retención (1.012 min) y el análisis espectral mostrado por las muestras y el estándar de Delfinidina-3-rutinoside, la antocianina cuantificada en el extracto y en los polvos microencapsulados podría ser Delfinidina-3-rutinoside. Todaro et al. (2009) identificaron en cáscara de berenjena, tres tipos de antocianinas, dentro de las que están delfinidina-3-rutinósido y las otras dos (6\% de la superficie total de los picos) no fueron identificadas. Asimismo Wu y Prior (2005) identificaron cuatro tipos de delfinidina en cáscara de berenjena, dentro de las que se encontraron delfinidina 3-rutinósido-5-galactósido, delfinidina 3-rutinósido-5-glucósido, delfinidina 3glucósido y delfinidina 3-rutinósido, siendo esta última la que se encontraba en mayor proporción.

Los valores medios de retención de antocianinas en los polvos microencapsulados con maltodextrina presentaron diferencias significativas $(P<0,05)$, para el factor concentración de agente encapsulante (maltodextrina). Se observaron los mayores porcentajes de retención de antocianinas en las microcápsulas con $30 \%$ de maltodextrina en el flujo de alimentación. Los porcentajes de 15 y $20 \%$ de sólidos en la alimentación no mostraron una influencia significativa en cuanto al porcentaje de retención del pigmento. Silva et al. (2013) reportaron la óptima retención de antocianinas al emplear maltodextrina al $30 \%$ a una temperatura de entrada del aire de secado de $180^{\circ} \mathrm{C}$. Tonon et al. (2010) encontraron que la maltodextrina por ser un material altamente soluble, forma partículas huecas durante el proceso de secado en el que la corteza es una matriz que contiene tanto el agente portador y el compuesto atrapado. 

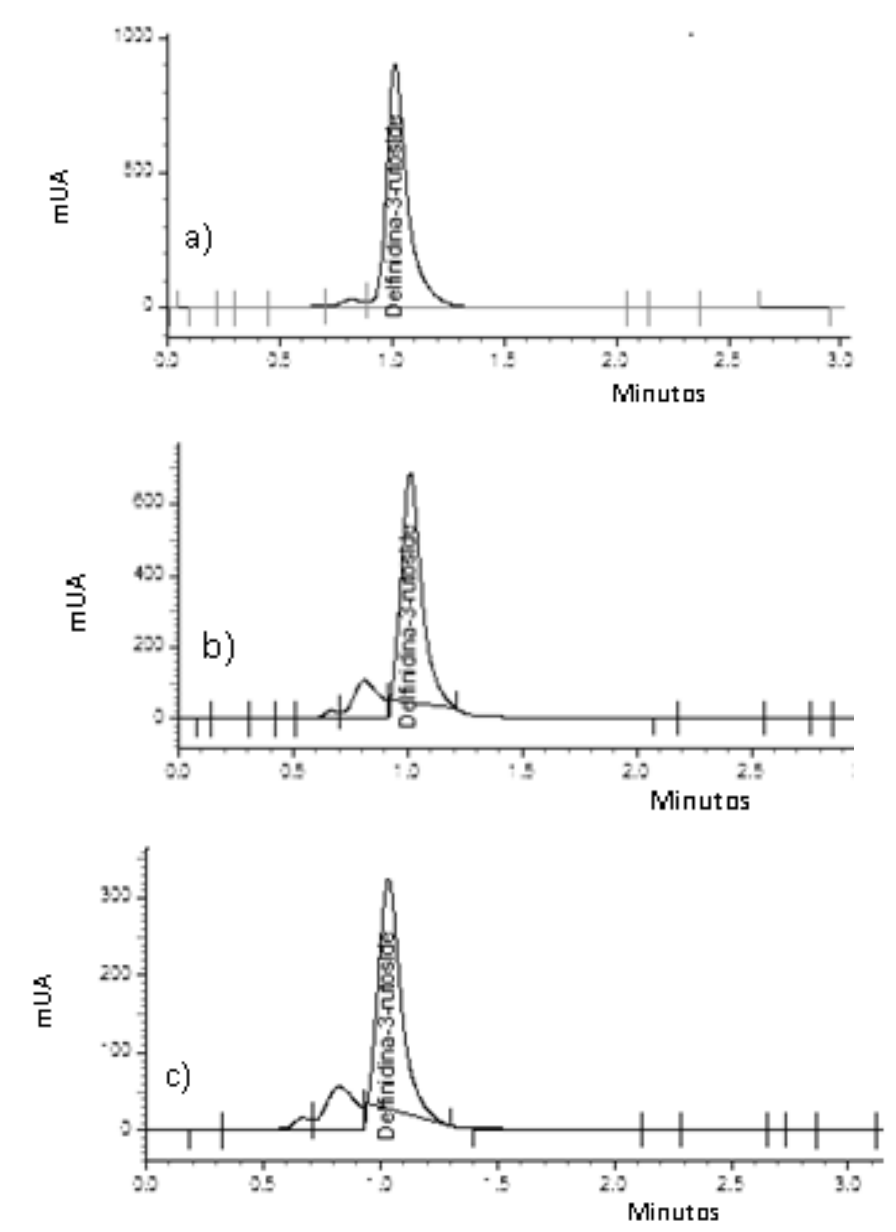

Fig. 2. Cromatograma $520 \mathrm{~nm}$ : a) estándar Delfinidina-3rutinoside b) Extracto de antocianinas y c) Extracto en polvo Microencapsulado a $180^{\circ} \mathrm{C}$ y $30 \%$ maltodextrina.

Por otro lado la temperatura de entrada del aire de secado no influyó significativamente en el porcentaje de retención de antocianinas de las microcápsulas. Esto puede deberse a que los tiempos de residencia empleados en el secado por atomización son pequeños, por lo que no alcanzaría a degradar los antocianos presentes. Bakowska-Barczak y Kolodziejczykb (2011) observaron que la temperatura de entrada del secado por debajo de $180^{\circ} \mathrm{C}$ no afectó significativamente el contenido de antocianinas en los polvos de grosella negra. Silva et al. (2013) manifestaron que el porcentaje de retención de antocianinas fue alto incluso cuando las temperaturas de salida eran altas, indicando que la temperatura óptima para obtener polvos con alta retención de pigmento, fue de $180^{\circ} \mathrm{C}$. Sin embargo, en este trabajo se encontró que si existe una diferencia significativa en la interacción de las dos variables independientes en estudio, pues, el mayor porcentaje de retención del pigmento $(98.4 \%)$ se obtuvo al emplear $30 \%$ de maltodextrina y $170{ }^{\circ} \mathrm{C}$ (fig. 1f).

Otros autores como Ersus y Yurdagel (2007) observaron que para sistemas que contienen maltodextrina con DE (equivalente dextrosa) menores a $29^{\circ} \mathrm{C}$ la temperatura de entrada del aire podría ser hasta $180^{\circ} \mathrm{C}$, al secar por aspersión antocianinas de zanahoria negra. Sin embargo Tonon et al. (2008) reportaron en su estudio que la temperatura del aire de entrada fue la única variable que afecta a la retención de antocianina. Los autores concluyen que el aumento de la temperatura de entrada de aire (150 a $202{ }^{\circ} \mathrm{C}$ ) condujo a un aumento en la pérdida de antocianinas, que es debido a la alta sensibilidad de estos pigmentos a las altas temperaturas. Cai y Corke (2000) pusieron de manifiesto que el aumento de temperatura de entrada de aire causa más pérdidas de pigmento y que temperaturas mayores a $180^{\circ} \mathrm{C}$ no son adecuadas para el secado por pulverización de betacianinas. En este estudio el porcentaje de retención de antocianinas fue alto en todas las condiciones experimentales con valores por encima del $85 \%$, lo cual es importante en términos de producción industrial del pigmento. Valores similares de porcentaje de retención de antocianinas han sido reportado por otros autores, Tonon et al. (2008) (77 al $86 \%$ ), Fang y Bhandari (2011) (94\%) en extractos de frutos de Açaí, Arrayán. Asimismo Saénz et al. (2009) reportaron porcentajes de retención de betacianina e indicaxantina por encima de $98 \%$ y $92 \%$, respectivamente. 


\section{Microscopia electrónica de barrido (SEM) de polvos.}

En la figura 3 , se puede observar la morfología de las microcápsulas a concentraciones de agente encapsulante de $30 \%$ con temperatura de 170 y $180{ }^{\circ} \mathrm{C}$. Las microcápsulas son de tamaño y forma variable, es decir hay capsulas de forma esférica con una superficie lisa y capsulas con superficie abollada o irregular. En la figura 3a y $3 d$, se puede observar que hay mayor cantidad de microcápsulas redondas y de superficie lisa al emplear la temperatura de aire de secado de $180^{\circ} \mathrm{C}$ que a la temperatura de $170^{\circ} \mathrm{C}$.

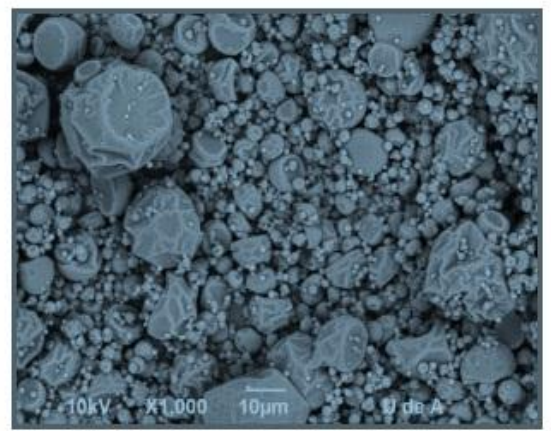

a)

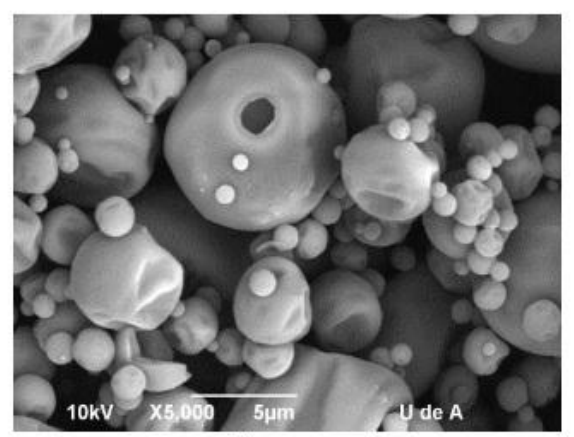

d)

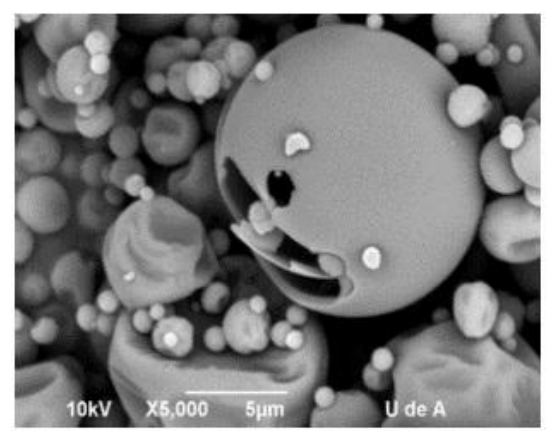

b)

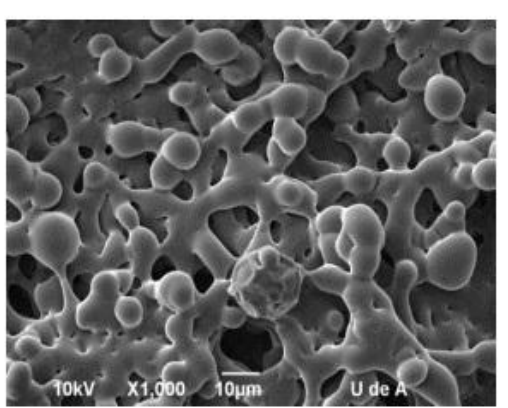

(e)

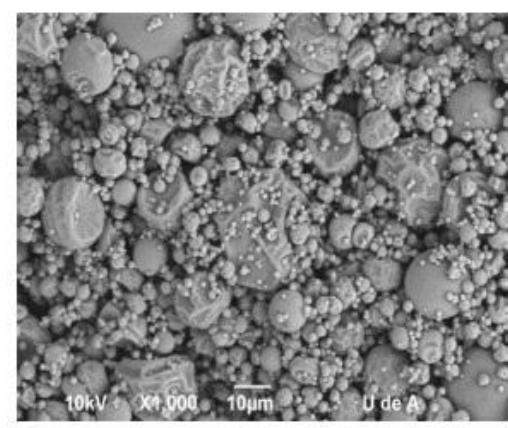

c)

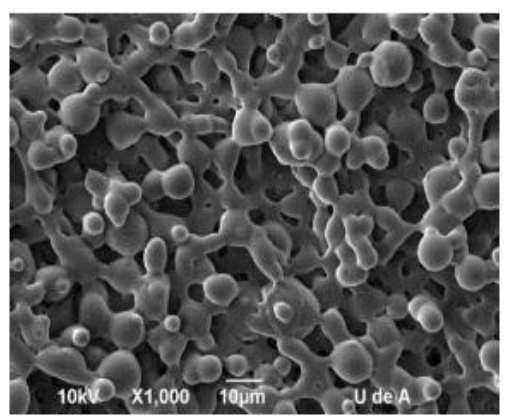

f)

Fig. 3. Fotografías de Microscopía Electrónica de Barrido (SEM) de microcápsulas a) $30 \% \mathrm{MD}$ y $170{ }^{\circ} \mathrm{C}$, $1000 x$. b) $30 \% \mathrm{MD}$ y $170{ }^{\circ} \mathrm{C} 5000 \mathrm{x}$. c) $30 \% \mathrm{MD}$ y $180{ }^{\circ} \mathrm{C}, 1000 \mathrm{x}$. d) $30 \% \mathrm{MD}$ y $180{ }^{\circ} \mathrm{C}, 5000 x$. e) $20 \%$ $\mathrm{MD}$ y $180^{\circ} \mathrm{C}, 1000 \mathrm{x}$. f) $20 \% \mathrm{MD}$ y $170^{\circ} \mathrm{C}, 1000 x$. Fuente: Autor

Esta estructura lisa y esférica de microencapsulados con maltodextrina ha sido observada por autores como Tonon et al. (2008); Escalona, (2004); Caparino et al. (2012); Ersus y Yurdagel (2007) en sus estudios de microencapsulación por secado aspersión empleando maltodextrinas en extractos de Acay, luteinaenocianina, mango y pigmentos zanahoria negra (Daucuscarota $L$ ) respectivamente. Asimismo Olaya et al. (2009) reportaron partículas que exhibían superficie lisa, y otras que presentaban superficies porosas con abolladuras superficiales en la encapsulación con maltodextrina de pigmentos de antocianos de Mora de Castilla (Rubus glaucus) y tamarillo (Solanum betaceum). La formación de abolladuras en la superficie de la capsula, generalmente puede ser atribuida a la contracción de las partículas durante el secado, debido a la drástica pérdida de humedad seguido de enfriamiento (Escalona, 2004; Sáenz et al., 2009; Silva et al., 2013). Por su parte Cai y Corke (2000) encontró que los equivalentes de dextrosa (DE) de la maltodextrina empleada también influye en la presencia de grietas y hendiduras en la superficie de la capsula, siendo la maltodextrina de bajos DE la que presenta mayor irregularidades y grietas superficiales.

Tonon et al. (2008) en su estudio de microencapsulado de Acai (Euterpe oleraceae Mart.) con maltodextrina, manifestaron que cuando la temperatura del aire de entrada fue baja, la mayoría de las partículas mostraron una superficie arrugada, y cuando aumentó la temperatura de secado obtuvo un mayor número de partículas con superficie lisa. Esto está relacionado con las diferencias en la velocidad de secado, que es más alta para las temperaturas elevadas, causando una evaporación más rápida del agua y que conduce a la formación de una costra lisa y dura. Las partículas con superficies rugosas pueden tener problemas en sus propiedades de flujo, además sus grandes áreas de contacto pueden hacerlas más susceptibles a reacciones de degradación, tales como la oxidación (Silva et al., 2013). En las figuras 3e y 3f, se muestra la morfología de las microcápsulas obtenidas con $20 \%$ de maltodextrina, en estas se observan unas microcápsulas apiladas y unidas entre sí. Esto podría ser debido a que la menor concentración de maltodextrina produjo una mayor hidratación de los polvos. 


\section{Rendimiento del proceso de Microencapsulación por secado por aspersión}

El rendimiento del proceso de secado por atomización varió significativamente en cuanto al factor temperatura y concentración de agente encapsulante, asimismo la interacción de las variables también fue significativa. El rendimiento más alto $(90.74 \%)$ se obtuvo con concentración de maltodextrina del $30 \%$ y una temperatura de secado de $180{ }^{\circ} \mathrm{C}$. Bakowska-Barczak y Kolodziejczykb (2011) obtuvieron el rendimiento más alto (86 \%) con maltodextrina DE 11 y temperatura de $150{ }^{\circ} \mathrm{C}$ mientras que para el mismo tipo de agente encapsulante el rendimiento fue de 72.6 y $55.1 \%$ para las temperaturas de 180 y $205{ }^{\circ} \mathrm{C}$ respectivamente. En los tratamientos realizados con $15 \%$ de maltodextrina se observó la adherencia del polvo encapsulado en las paredes de la cámara del secador y por consiguiente la pérdida de material encapsulado siendo los tratamientos a $30 \%$ de maltodextrina los que tuvieron una menor pérdida de material por adhesión. Valduga et al. (2008) relacionaron posiblemente a que esta adherencia puede estar relacionada con el alto contenido de extracto en el flujo de alimentación, el cual podría contener algunos compuestos responsables de este comportamiento.

En este estudio el rendimiento del proceso aumentó con el incremento en la temperatura del aire de entrada. Esto es debido a que las altas temperaturas de entrada, dan mayor eficiencia de calor y mejoran los proceso de transferencia de masa, lo cual conduce a un rendimiento más alto del proceso (Tonon et al., 2008; Tee et al., 2012). Los rendimientos bajos del $26.06 \%$ se obtuvieron con $15 \%$ de maltodextrina y 170 ${ }^{\circ} \mathrm{C}$. Las pérdidas de polvo durante el secado por pulverización asociada al bajo rendimiento se debieron principalmente a la aglomeración de algunas partículas del polvo que se deposita sobre la pared de la cámara secadora, y en el ciclón. Fang y Bhandari (2011) encontraron rendimientos del polvo de extractos de arrayán secado por aspersión del $55 \pm 3 \%$, cuando empleó maltodextrina con sólidos ajustados hasta $11^{\circ}$ brix y temperatura de entrada de $150{ }^{\circ} \mathrm{C}$. Sin embargo Tonon et al. (2008) observaron un efecto negativo de la concentración de maltodextrina en el rendimiento del proceso, probablemente debido a la viscosidad de la mezcla, que se incrementó exponencialmente con esta variable; este aumento de la viscosidad de alimentación puede causar que los sólidos se adhieren en la pared de la cámara principal, lo que podría reducir el rendimiento del proceso.

\section{Capacidad antioxidante de polvos microencapsulados}

En la tabla 1, se puede observar los valores de IC50, TEAC (Capacidad antioxidante equivalente de Trolox) y VEAC, (Capacidad antioxidante equivalente de ácido ascórbico) estos dos últimos calculados con base a las curvas patrones de los reactivos de Trolox y Ácido ascórbico. Curva calibrado ácido ascórbico $\mathrm{Y}=\mathrm{m}$ a+ $b, a=19.098$ y $b=9.775$.

Tabla 1. Análisis de capacidad antioxidante para los polvos microencapsulados

\begin{tabular}{lccccc}
\hline Tratamiento & $\mathrm{b}$ & $\mathrm{R}^{2}$ & $\mathrm{IC} 50^{*}$ & TEAC & VEAC \\
\hline $170{ }^{\circ} \mathrm{C}-15 \%$ & 0,82 & 0,97 & 52,18 & 241,10 & 228,10 \\
$170{ }^{\circ} \mathrm{C}-20 \%$ & 0,79 & 0,98 & 67,92 & 185,20 & 175,20 \\
$170{ }^{\circ} \mathrm{C}-30 \%$ & 0,41 & 0,97 & 123,54 & 101,80 & 96,30 \\
$180{ }^{\circ} \mathrm{C}-15 \%$ & 0,89 & 0,94 & 49,76 & 252,80 & 239,10 \\
$180{ }^{\circ} \mathrm{C}-20 \%$ & 0,76 & 0,98 & 70,09 & 179,50 & 169,70 \\
$180{ }^{\circ} \mathrm{C}-30 \%$ & 0,41 & 0,97 & 122,06 & 103,10 & 97,49 \\
\hline
\end{tabular}

Para el cálculo de los IC50 de cada uno de los polvos, se llevó a cabo una regresión lineal, cuyos $\mathrm{R}^{2}$, están por encima de 0.90 , lo que indica una buena relación entre la concentración de los polvos y la actividad inhibitoria del radical. El factor temperatura no tuvo una influencia significativa en el IC50 de los polvos microencapsulados, mientras que el factor porcentaje de maltodextrina si la presentó. Asimismo la interacción entre las dos variables no presentó una influencia significativa. De esta manera los tratamientos a $170{ }^{\circ} \mathrm{C}$ y $180^{\circ} \mathrm{C}$, exhibieron los mayores valores de IC50 (Tabla 1) y por lo tanto, un valor de TEAC y VEAC menor. Bakowska-Barczak y Kolodziejczyk (2011) observaron que no hubo diferencias significativas en la capacidad antioxidante de los polvos al emplear maltodextrinas durante el secado por aspersión de Jaboticaba, reportando valores de actividad antioxidante de 8.3 a $9.1 \mathrm{mM} / 100 \mathrm{~g}$ polvo.

Los tratamientos con menor porcentaje de maltodextrina presentaron menores IC50, de esta manera el tratamiento a $180{ }^{\circ} \mathrm{C}$ y $15 \%$ de maltodextrina mostró altos valores de TEAC y VEAC, equivalentes 252.8 y $239.1 \mu \mathrm{M}$ de Trolox y Ácido ascórbico por cada gramo de polvo respectivamente. Lo anterior indica que este tratamiento presentó la mayor actividad antioxidante frente al radical ABTS*. .Ersus y Yurdagel (2007) encontraron una relación negativa entre el contenido de antocianinas de los polvos y el IC50 de los mismos, expresando que el aumento en el contenido de antocianinas en los polvos, produjo una disminución de los IC50 y por ende una mayor capacidad antioxidante. 


\section{Análisis de color de los polvos secados por atomización}

Los resultados del análisis de color de los polvos microencapsulados se resume en la tabla 2. En relación con el índice de $L^{*}$, que mide luminosidad (blanco / negro), el análisis estadísticos muestra que los principales efectos temperatura y concentración de agente encapsulante presentan diferencia significativa $(p$ $<0,05)$, así como el efecto de interacción entre las variables. En el análisis de los datos se encontró que los valores más bajos de $\mathrm{L}^{*}$, corresponden al tratamiento con $15 \%$ de maltodextrina y $170{ }^{\circ} \mathrm{C}$, el cual presenta una apariencia de un color rosado oscuro y los valores más altos de $L^{*}$ fueron con $30 \%$ de sólidos en la alimentación que significan un color claro. Un comportamiento similar observó Valduga et al. (2008) al evaluar el índice $L^{*}$ en polvos de extractos de antocianinas de Uva, en donde el índice $L^{*}$ fue más bajo (49.4) en el tratamiento con mayor volumen de extracto.

Tabla 2. Parámetros de color para polvos microencapsulados por secado por atomización.

\begin{tabular}{|c|c|c|c|c|c|}
\hline \multirow[t]{2}{*}{ Tratamiento } & \multicolumn{5}{|c|}{ Parámetros de color } \\
\hline & $\mathrm{L}^{*}$ & $\mathrm{a}^{*}$ & $\mathrm{~b}^{*}$ & $\mathrm{C}^{*}$ & ${ }^{\circ} \mathrm{H}$ \\
\hline $170{ }^{\circ} \mathrm{C}-15 \%$ & $41,0 \pm 0,0$ & $39,9 \pm 0,0$ & $5,3 \pm 0,01$ & $40,3 \pm 0,01$ & $7,6 \pm 0,01$ \\
\hline $170{ }^{\circ} \mathrm{C}-20 \%$ & $53,9 \pm 1,1$ & $35,9 \pm 0,8$ & $1,9 \pm 0,40$ & $36,0 \pm 0,83$ & $3,0 \pm 0,62$ \\
\hline $170{ }^{\circ} \mathrm{C}-30 \%$ & $56,2 \pm 0,0$ & $37,3 \pm 0,0$ & $1,8 \pm 0,01$ & $37,3 \pm 0,01$ & $2,8 \pm 0,01$ \\
\hline $180{ }^{\circ} \mathrm{C}-15 \%$ & $46,3 \pm 0,9$ & $38,7 \pm 0,8$ & $5,3 \pm 0,49$ & $39,0 \pm 0,93$ & $7,7 \pm 0,55$ \\
\hline $180{ }^{\circ} \mathrm{C}-20 \%$ & $52,9 \pm 0,5$ & $35,9 \pm 0,8$ & $2,4 \pm 0,04$ & $35,5 \pm 0,85$ & $3,9 \pm 0,17$ \\
\hline $180{ }^{\circ} \mathrm{C}-30 \%$ & $56,0 \pm 0,0$ & $37,3 \pm 0,0$ & $2,6 \pm 0,05$ & $37,4 \pm 0,05$ & $3,9 \pm 0,00$ \\
\hline
\end{tabular}

Investigadores como Caparino et al. (2012); Ahmed et al. (2010) observaron los más altos valores de L*, al adicionar maltodextrina en la obtención de polvos de mango y batata morada mediante secado en spray respectivamente. (Los valores de índices de $L^{*}$, son similares a los reportados por autores como Cai y Corke (2000) (49.35) en polvos de betacianinas. Los tratamientos con $15 \%$ de maltodextrina presentaron un color más brillante, con más altos valores $\mathrm{a}^{*}$, lo que refleja el mayor contenido de antocianinas de estos polvos. Se observa que a medida que aumenta el volumen de extracto, y mayor contenido del pigmento mayor será el índice $a^{*}$. Otros autores observan comportamiento similar (Valduga et al. 2008; Sáenz et al. 2009). Las diferencias en la concentración de maltodextrina tuvo efectos significativos $(p<0,05)$ en los valores de $C^{*}$, es decir, con la disminución de la concentración de maltodextrina los valores de $C^{*}$ de los polvos se incrementaron. Sin embargo la temperatura y el efecto de la interacción no fueron significativos. Los valores de ${ }^{\circ} \mathrm{H}$ fueron diferentes estadísticamente con respecto al factor temperatura y al factor concen-tración de maltodextrinas, siendo la concentración al $15 \%$ la que presentó mayor ángulo ${ }^{\circ} \mathrm{H}$ y las del 20 y $30 \%$ no difirieron entre sí. Los valores ${ }^{\circ} \mathrm{H}$ fueron altos, indicando que son muy cercanos al color rojo. Silva et al. (2010) verificaron que extractos de jaboticaba microencapsulado con $30 \%$ de maltodex-trina, tenían valores $\mathrm{C}^{*}$ y ${ }^{\circ} \mathrm{H}$ de 22.2 y 0.18 respectivamente. Asimismo Caparino et al. (2012) reportó ángulo de tono $\left({ }^{\circ} \mathrm{H}\right)$ altos y valores croma bajos indicando un color opaco en polvos de mango secos por aspersión.

\section{CONCLUSIONES}

Los resultados obtenidos para Los factores de extracción de antocianinas, tiempo, temperatura y concentración de solvente, muestran mejores condiciones con $53 \%$ de solvente (Etanol), tiempo de 3 horas y temperatura de $29^{\circ} \mathrm{C}$ con contenido de antocianina de $115 \mathrm{mg} / 100 \mathrm{~g}$ en cáscara de berenjena. Tiempos prolongados de extracción del pigmento y temperaturas altas, causan una disminución del contenido de antocianinas en los extractos, lo que demuestra su alta sensibilidad a los tratamientos de extracción severos. La temperatura de entrada del aire del secador por aspersión y la concentración del agente encapsulante influyó significativamente $(P<0,05)$, en las variables fisicoquímicas de humedad, actividad de agua y solubilidad de los extractos microencapsulados. Los pigmentos microencapsulados mediante el secado por aspersión con $30 \%$ de maltodextrina y $180^{\circ} \mathrm{C}$ presentaron buenas propiedades fisicoquímicas, con menores contenidos de humedad (3.43\%) y actividad de agua $(0.26)$ y mayores porcentajes de solubilidad $(93.61 \%)$. De acuerdo a los tiempos de retención $(1.012 \mathrm{~min})$ y el análisis espectral mostrado por las muestras y el estándar de Delfinidina-3-rutinoside, la antocianina cuantificada en el extracto y en los polvos microencapsulados podría ser Delfinidina-3-rutinosido. Los polvos microencapsulados presentaron parámetros de color ${ }^{\circ} \mathrm{H}$ entre 2 y $7^{\circ}$, lo que indica un color rojo con alta tonalidad, siendo el tratamiento con $15 \%$ de maltodextrina el que presentó los mayores valores de tonalidad $\left(7^{\circ} \mathrm{H}\right)$.

\section{REFERENCIAS}

Aguilera-Ortíz, M., M. Reza-Vargas, R. Chew J. Aguilar y P. Ramírez, Antocianinas de higo como colorantes para yogur natural. Revista de Ciencias Biológicas y de la Salud, XIV (1): 18-24 (2012). 
Ahmed, M., M. Sorifa y J. Eun, Impact of $\alpha$-amylase andmaltodextrin on physicochemical, functional and antioxidant capacity of spray-dried purple sweet potato flour. Journal Science Food Agricultural 90: 494-502 (2010).

Bakowska-Barczaka, A. M. y P. P. Kolodziejczykb, Black currant polyphenols: Their storage stability and microencapsulation. Industrial Crops and Products 34: 1301- 1309 (2011).

Cai, Y. y H Corke, Production and Properties of Spray-dried Amaranthus Betacyanin Pigments. Journal Of Food Science 65 (6): 1248 -1252 (2000)

Calogero, G. y G. Di Marco, Red Sicilian orange and purple eggplant fruits as natural sensitizers for dyesensitized solar cells. Solar Energy Materials \& Solar Cells, 92: 1341- 1346 (2008)

Cano-Chauca, M., P. Stringheta, L. Sardagna y J. Cal-Vidal, Mango juice dehydration spray drying using different carriers and functional characterization. Proceedings of the 14th International Drying Symposium, São Paulo, Brazil, C: 2005-2012 (2004).

Caparino, O., J. Tang, C. Nindo, S. Sablani, J. Powers y J. Fellman, Effect of drying methods on the physical properties and microstructures of mango (Philippine 'Carabao'var.) powder. Journal of Food Engineering 111: 135-148 (2012).

Castañeda, A., M. Pacheco, M. Páez, J. Rodríguez y C. Galán, Chemical studies of anthocyanins: A review. Journal Food Chemistry 113: 859-871 (2009).

Ceballos, A., Estudio comparativo de tres sistemas de secado para la producción de un polvo deshidratado de frutas. Tesis para optar Magister en Ingeniería química. Universidad Nacional. Manizales, Colombia. (2008).

Da Silva, G., P. Constant, R. Figueiredo y S. Moura, Formulação e estabilidade de corantes de antocianinas extraídas das cascas de Jabuticaba (Myrciaria ssp.). Alim. Nutr., Araraquara, 21 (3) : $429-436$ (2010).

Durst, R. y R. Wrolstad, Separation and Characterization of Anthocyanins by HPLC. Current Protocols in Food Analytical Chemistry. F1.3- F1.3.13 (2001).

Ersus S. y U. Yurdagel, Microencapsulation of anthocyanin pigments of black carrot (Daucuscarota L.) by spray drier. Journal Food Engineering. 80: 805-812 (2007).

Escalona, S., Encapsulados de luteina-enocianina y su aplicación en alimentos. Tesis Ingeniero en Alimentos. Universidad de Chile. Santiago de Chile (2004).

Fang, Z. y B. Bhandari, Effect of spray drying and storage on the stability of bayberry polyphenols. Food Chemistry 129: 1139-1147 (2011)

FAO. 2007. Berenjena (Solanum melongena L). Disponible en URL: http://www.fao.org/inpho/content/documents/vlibrary/ae620s/Pfrescos/berenjena.htm. 23 mayo de (2010).

García, E., E. Hernández, C. De Paula y H. Aramendiz, Caracterización bromatológica de la berenjena (Solanum melongena L.) en el departamento de Córdoba. Revista Temas Agrarios 8 (1): $27-32$ (2003).

García, G., M. González, H. Ochoa y Medrano, Microencapsulación del jugo de cebada verde mediante secado por aspersión. Revista Ciencia y Tecnología Alimentaria 4(4): 262-266 (2004).

Garzón, G., Las antocianinas como colorantes naturales y compuestos bioactivos: revisión. Acta Biológica Colombiana 13 (3): 27 - 36. (2008).

Ichiyanagi, T., N. Terahara, M. Rahman y T. Konishi, Gastrointestinal uptake of nasunin, acylated anthocyanin in eggplant. Journal of Agricultural and Food Chemistry, 54: (15): 5306-5312 (2006).

Jittanit, W., M. Chantara-In, T. Deying y W. Ratanavong, Production of tamarind powder by drum dryer using maltodextrin and Arabic gum as adjuncts. Songklanakarin Journal Science. Technology 33 (1): 33-41 (2011).

Longo, L. y G. Vasapollo, Extraction and identification of anthocyanins from Smilax aspera L. berries. Food Chemistry 94: 226-231 (2004).

Martínez, B., Evaluación de la estabilidad, las propiedades antioxidantes y antimicrobianas de dos extractos de plantas aromáticas limoncillo (Cymbopogon citratus) y cúrcuma (Curcuma longa).Tesis Ingeniero de Alimentos. Universidad de Córdoba. Colombia (2009).

Miravet, V. G., Secado por Atomización de zumo de granada. Tesis Master en Ingeniería Ambiental y Procesos Químicos y Biotecnológicos. Universidad Politécnica de Cartagena. Cartagena Colombia (2009).

Nisha, P., P. Nazar y P. Jayamurthy, A comparative study on antioxidant activities of different varieties of Solanum melongena. Food and Chemical Toxicology 47: 2640-2644 (2009). 
Noda, Y., T. Kneyuki, K. Igarashi, A. Mori y L. Packer, Antioxidant activity of nasunin, an anthocyanin in eggplant peels. Toxicology 148: 119-123 (2000).

Ochoa, L., S. González, J. Morales, N. Rocha, N., Trancoso y M. Urbina, Propiedades de rehidratación y funcionales de un producto en polvo a base de jugo de granada y manzana. Ciencia@Uaq. 4(2):19-25 (2011)

Olaya, C., M. Castaño y G. Garzón, Stability of anthocyanins from Rubus glaucus Benth and Solanum betaceum Cav.dark-red strain as affected by temperature, storage time and water activity. Acta Biológica Colombiana 14 (3): 141-156 (2009).

Owusu, A., Chemistry Postharvest. En: Introduction to Food Chemistry. Primera Edición. Editorial CRC Press. United States of America. P. 219 (2005).

Phisut, N., Spray drying technique of fruit juice powder: some factors influencing the properties of product. International Food Research Journal 19 (4): 1297-1306 (2012).

Poo, B., Concentración de Antocianinas en Jugo de Cranberries (Vaccinium macrocarpon Ait.) mediante Nanofiltración. Tesis de Licenciado en Ciencias de los Alimentos. Universidad Austral de Chile. Chile (2005).

Provenzi, G., L. Falcão y R. Fett, R. Estabilidade de Antocianinas de Uvas Cabernet Sauvignon com $\beta$ - e $\gamma^{-}$ Ciclodextrinas. Brazil Journal Food Technology. 9 (3): 165-170 (2006).

Saénz, C., S. Tapia, J. Chávez y P. Robert, Microencapsulation by spray drying of bioactive compounds from cactus pear (Opuntia ficus-indica). Food Chemistry 114: 616-622 (2009).

Silva, P., P. Stringheta, R. Teófilo y I. Nolasco, Parameter optimization for spray-drying microencapsulation of jaboticaba (Myrciaria jaboticaba) peel extracts using simultaneous analysis of responses. Journal of Food Engineering. artículo en impresión (2013).

Silva, M., H. Rogez y Y. Larondelle, Optimization of extraction of phenolics from Inga edulis leaves using response surface methodology. Separation and Purification Technology 55 (3): $381-387$ (2010).

Singh, A., D. Luthria, T. Wilson, N. Vorsa, V. Singh, G. Banuelos y S. Pasakdee, Polyphenols content and antioxidant capacity of eggplant pulp. Food Chemistry 114: 955-961 (2009).

Ribeiro, F. y P. Stringheta, Microencapsulamento de antocianinas. Biotecnologia Ciência e Desenvolvimento 36: (2006).

Rivas, C., Microencapsulacion y estabilizacion enzimatica del jugo de chirimoya. Tesis para obtener título de Maestría en Ciencias en Bioprocesos. Instituto Politécnico Nacional. México D. F. (2010).

Ruiz-Cabrera, M., L. Espinosa, C. Aviles, R. González, M. Moscosa, A. Grajales y M. Abud, Spray-Drying of Passion Fruit Juice Using Lactose-Maltodextrin Blends as the Support Material. Braz. Arch. Biol. Technol. 52 (4): 1011-1018 (2009).

Tee, L. H., C. A. Luqman, K. Y. Pin, R. A. Abdull y Y. A. Yusof, Optimization of spray drying process parameters of Piper betle $L$. (Sirih) leaves extract coated with maltodextrin. Journal of Chemical and Pharmaceutical Research, 4 (3):1833-1841 (2012).

Todaro, A., F. Cimino, P. Rapisarda, A. Catalano, R. Barbagallo y G. Spagna, Recovery of anthocyanins from eggplant peel. Journal Food Chemistry 114: 434-439 (2009).

Tonon, R., S. Freitas y M. Hubinger, Spray drying of acai (Euterpe oleraceae mart.) juice: effect of inlet air temperature and type of carrier agent. Journal of Food Processing and Preservation, 35 (5): 691-700 (2011).

Tonon, R., C. Brabet y M. Hubinger, Anthocyanin stability and antioxidant activity of spray-dried açai (Euterpe oleracea Mart.) juice produced with different carrier agents. Food Research International, 43 (3): 907-914 (2010).

Tonon, R., C. Brabet y M. Hubinger, Influence of process conditions on the physicochemical properties of acai (Euterpe oleraceae Mart.) powder produced by spray drying. Journal of Food Engineering 88: 411-418 (2008).

Valduga, E., L. Lima, R. Prado, F. Ferreira y H. Treichel, Extração, secagem por atomização e microencapsulamento de antocianinas do bagaço da uva isabel (vitis labrusca). Ciênc. Agrotec. 32 (5): 1568-1574 (2008).

Wu, X. y R. Prior, Identification and characterization of anthocyanins by high-performance liquid chromatography-electrospray ionization-tandem mass spectrometry in common foods in the united states: Vegetables, nuts, and grains. Journal of Agriculture and Food Chemistry, 53: 3101-3113 (2005). 